\title{
A novel low-cost smart leaf wetness sensor
}

\author{
Gemma Hornero*, Jorge E. Gaitán-Pitre, Ernesto Serrano-Finetti, Oscar Casas, Ramon Pallas-Areny
}

\section{A R T ICLE INFO}

\section{Keywords:}

Leaf wetness sensor

Capacitive sensor

Charge-transfer circuit

Precision agriculture

Plant disease

\begin{abstract}
A B S T R A C T
Foliar wetness plays an essential role in plant disease cycles and epidemic development yet no cost-effective leaf wetness sensors (LWSs) are available that could be deployed within large areas to better understand that role. Electronic LWSs comprise an artificial leaf and the electronic circuitry able to measure electrical impedance changes due to water film or drops on the leaf surface. We propose a simple, compact and low-cost electronic interface circuit (EIC) for artificial leaves based on capacitance changes. The circuit relies on the charge-transfer capacitive sensing method and it is implemented by a microcontroller unit (MCU), which offers computation and communication capabilities currently missing in commercial LWSs, This EIC can be used in custom and commercial artificial leaves hence suits studies that require a close emulation of particular plant leaves.
\end{abstract}

\section{Introduction}

Between 2003 and 2012, about 12.5 million hectares were allegedly disturbed by plant diseases, mostly in Asia and Europe (Lierop et al., 2015). To mitigate this problem, several disease-warning systems have been developed that rely on mathematical, empirical or hybrid models that need leaf wetness duration data (LWD) (Rowlandson et al., 2015). Leaf wetness can result from dew, fog, rain, and overhead irrigation. It is a meteorological variable with no formal definition neither there is any recommended method to measure it (Madeira et al., 2002). This notwithstanding, electronic leaf wetness sensors (LWSs) are widely accepted and are the current technology of choice for LWD determination (Rowlandson et al., 2015). LWSs measure the change in the electrical impedance of a wire grid on the leaf, a clip attached to the leaf, or, more commonly, an artificial leaf (Sentelhas et al., 2004), and yield an output signal that changes according to the sensor's surface wetness. The dry/ wet thresholds to determine LWD from that output signal are mostly empirical (Wichink Kruit et al. 2004), so that current LWSs are intended to be connected to external devices such as data loggers for further data processing to determine LWD.

Artificial leaves are usually built from a rectangular-, circular- or oval-shaped electrical insulator such as an electronic printed circuit board (PCB) or a ceramic plate, and are based on either resistive or capacitive grids. Resistive artificial leaves use a grid built from two inter- digitated electrodes covered by hydrophilic material so that water accumulated on the surface, as drops or a film, reduces the electrical resistance between the electrodes (Fig. 1, left). Capacitive artificial leaves also use interdigitated electrodes but coated with dielectric material, hence water deposited on the surface affects the capacitance measured between the electrodes. Capacitive LWSs are more accurate and robust against surface contamination than their resistive counterparts but tend to be more expensive (Fig. 1, right). Further, leaf shapes and sizes available are limited and their cost, in excess of 100 USD, hinders their wide use in experimental studies that should involve many units, for example to assess the effect of sensor location and orientation, or in a disease-warning sensor network to be deployed within a wide area.

In this paper we describe a low-cost smart LWS that comprises an artificial leaf built on a PCB that emulates existing commercial models, and a novel electronic interface circuit (EIC) for capacitive sensors that is based on the charge-transfer capacitive sensing method and a low-end microcontroller unit (MCU). First, the sensitivity to different wetness conditions of the artificial leaves (custom and commercial) is assessed. Then, leaf wetness measurement performance is compared in two different setups: (i) using the novel EIC with the custom leaf design and with a commercial unit, and (ii) using the proposed smart LWS (electronic interface plus custom-designed artificial leaf) and a commercial LWS that includes an electronic interface with voltage output. Performance tests have been carried out in lab and outdoor conditions.

\footnotetext{
* Corresponding author.

Email address: gemma.hornero@upc.edu (G. Hornero)
} 

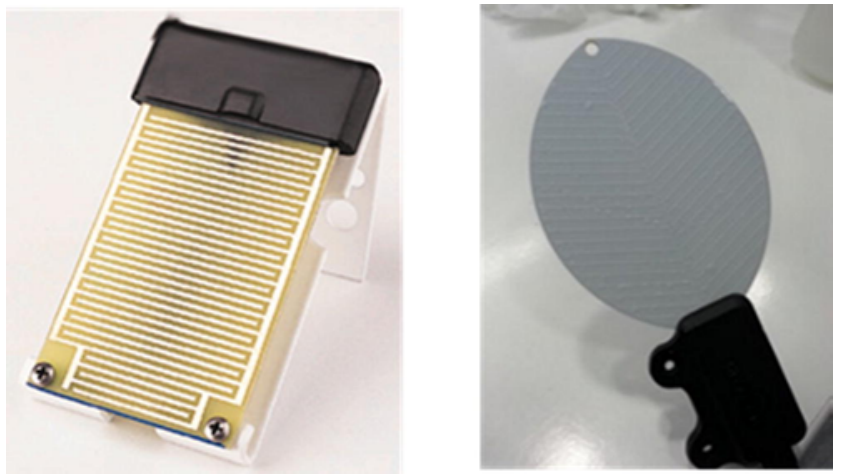

Fig. 1. LWS types: Resistive (left) (Davis Instruments) and Capacitive (right) (Decagon Devices).

\section{Development of a leaf wetness sensor}

\subsection{Capacitive artificial leaf}

We have designed two capacitive artificial leaves with rectangular (RECT) and oval (OVAL) shapes, printed on a $1.6 \mathrm{~mm}$ thick fiberglass plate, the usual PCB substrate, and whose dimensions are shown in Fig. 2. Capacitive leaf wetness sensors rely on the high value of the relative dielectric constant of water $\left(\varepsilon_{\mathrm{r}} \approx 80\right)$ with respect to fiberglass $\left(\varepsilon_{\mathrm{r}}\right.$ $\approx 3-6$ ). Thus, the capacitance between two electrodes underneath the "leaf" surface will depend on the presence of water drops or film on the top fiberglass surface and on the shape and dimensions of the sensor. Both sensors have interdigitated electrode patterns to increase their effective area hence the capacitance between them. The penetration depth of the electric fields above the interdigitated electrodes is proportional to the spacing between the centerlines of the sensing and the driver fingers (Mamishev et al., 2004).

The PCB electrode side is covered with a $25 \mu \mathrm{m}$ protective coating (Clear Protective Lacquer CPL200 H - Electrolube) while the other side is left unprotected. The coating protects the PCB substrate against humidity hence fulfilling one key requirement of electronic LWS (Lau et al., 2000). Electrode width and gap are different for each sensor: $2.5 \mathrm{~mm}$ and $1 \mathrm{~mm}$ for the RECT sensor (Fig. 2, left) and $0.4 \mathrm{~mm}$ and $0.4 \mathrm{~mm}$ for the OVAL sensor (Fig. 2, right).

The accuracy of electronic LWSs is mainly limited by their physical characteristics (material, dimensions and shape) and their location and spatial orientation when deployed. Commercial LWSs are designed to mimic the thermodynamic properties of a standard plant leaf whose specific heat is about $3750 \mathrm{~J} \mathrm{~kg}^{-1} \mathrm{~K}^{-1}$. Since average leaf density is about $0.95 \mathrm{~g} / \mathrm{cm}^{3}$ and its thickness is about $0.4 \mathrm{~mm}$, the leaf's heat capacity is about $1425 \mathrm{Jm}^{-2} \mathrm{~K}^{-1}$, that can be closely emulated by a $0.65 \mathrm{~mm}$ fiberglass plate whose heat capacity is $1480 \mathrm{Jm}^{-2} \mathrm{~K}^{-1}$
(Campbell Scientific). We have used a thicker plate because of availability hence our custom sensors will have a larger heat capacity that translates into dry/wet thresholds different from those of commercial sensors. The PCB coating effect on thermal conductivity is unknown because the coating's thermal properties are not specified.

To obtain results that truly represent events in a large area, LWSs must be placed in a suitable position because sun and wind exposure heavily affect dew deposition and evaporation hence the impedance measured. Schmitz and Grant (2009), for example, investigated the variability of dew duration in resistive sensors in a soybean canopy and found that there was a vertical gradient of wetness during dew events, and that wetness duration at the top of the canopy was longer for dew events than for rainfall events. In the middle of the canopy, the frequency of wetting was also higher for dew than rain, but wetness due to rain lasted twice than wetness due to dew. At the bottom of the canopy, wetness duration because of dew was rarely seen. Therefore, the deployment of several LWSs at different heights within the canopy could surely provide a more accurate picture of leaf wetness duration.

\subsection{Electronic interface circuit design}

Interface circuits for capacitive sensors are available wherein the sensor is directly connected to an MCU that converts capacitance to a digital value without any previous signal conditioning stage or analog-to-digital converter, which makes those circuits simple, compact, and low cost. They are based on the charge-transfer method (Philipp, 1999), where the unknown capacitance is calculated by counting the number of charge-transfer cycles needed to charge a reference capacitor to a threshold voltage via the capacitive sensor (Fig. 3, left). $C_{x}$ is the sensor capacitance and $C_{\mathrm{r}}$ is a reference capacitor whose value is selected to be much greater than $C_{x}$. Pins PX.0 and PX.1 are standard input/output digital pins configurable as inputs (high-impedance input), or as outputs that provide $V_{\mathrm{OL}}$ and $V_{\mathrm{OH}}$ output voltage levels that correspond to a digital " 0 " and " 1 " respectively. $V_{x}$ and $V_{\mathrm{r}}$ are the voltages across $C_{x}$ and $C_{r}$ respectively.

The unknown capacitance $C_{x}$ is measured in a three-stage sequence controlled by the MCU:

(1) Reset (only at the beginning of each new measurement cycle). PX.0 and PX.1 are both set as outputs that provide a digital " 0 " $\left(V_{\mathrm{OL}} \approx\right.$ $0 \mathrm{~V}$ ). Therefore, $C_{\mathrm{r}}$ is discharged towards $V_{\mathrm{OL}}$, so that $V_{\mathrm{r}}[0] \approx 0$.

(2) Charging stage. PX.0 changes to a digital " 1 " $\left(V_{\mathrm{OH}}\right)$ and PX.1 is set as an input port (high-impedance state). $C_{x}$ charges exponentially towards $V_{\mathrm{OH}}$ (Fig. 3, ascending lines).

(3) Charge-redistribution stage. PX.0 is now set as an input port while PX.1 is set as an output at $V_{\mathrm{OL}}$ so that charge redistributes between $C_{x}$ and $C_{\mathrm{r}} . V_{x}$ decreases and $V_{\mathrm{r}}$ increases (Fig. 3, rising lines). The control program starts to increment the number of charge-transfer cycles $N_{x}$ and goes back to the charging state. The charging and
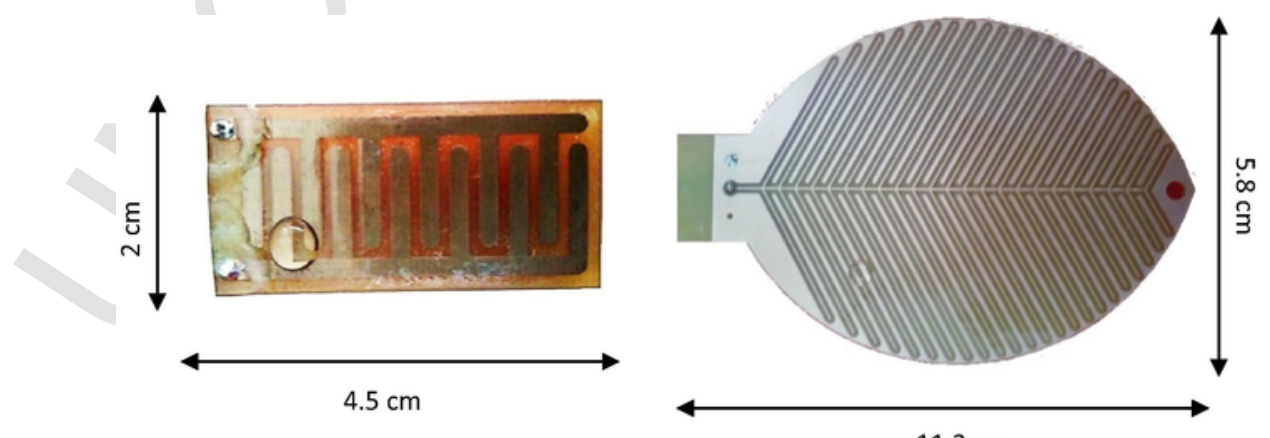

$11.2 \mathrm{~cm}$

Fig. 2. Custom RECT (left) and OVAL (right) capacitive leaf wetness sensors (not to scale). 

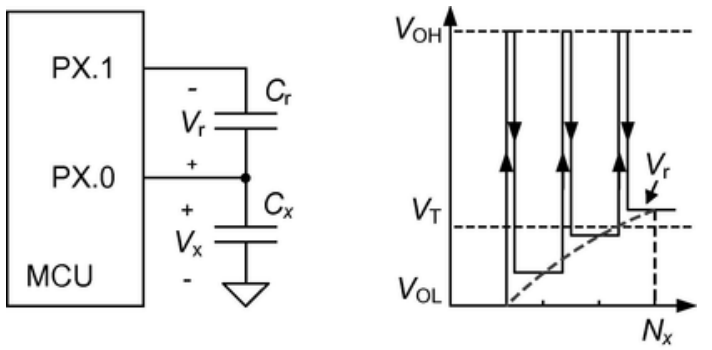

Fig. 3. Direct capacitive sensor-to microcontroller interface circuit and voltage across $C_{x}$ during a measurement cycle.

charge-redistribution stages are repeated until the voltage across $C_{\mathrm{r}}$ reaches the input threshold $V_{\mathrm{T}}$ at PX.0. Since $V_{\mathrm{r}}[0]=0$, after $N_{x}$ cycles the unknown capacitance can be approximated by (Fig. 3, right) (Gaitán-Pitre et al., 2009).

$C_{x} \approx \frac{C_{r}}{N_{x}} \ln \left[\frac{V_{\mathrm{OH}}}{V_{\mathrm{OH}}-V_{T}}\right]$.

$V_{\mathrm{OH}}$ and $V_{\mathrm{T}}$ depend on the supply voltage $\left(V_{\mathrm{DD}}\right)$ and ambient temperature.

The MCU selected to implement this interface was the PIC16F785 (Microchip Technology) operating at $4 \mathrm{MHz}$. For $V_{\mathrm{DD}}=2.50 \mathrm{~V}$, we measured $V_{\mathrm{OH}}=2.48 \mathrm{~V}$, and $V_{\mathrm{T}}=0.88 \mathrm{~V}$, which are within the voltage ranges specified by the manufacturer. These values are assumed to remain constant during the measurement time. If the circuit is calibrated by measuring two known capacitors, then the dependence on the supply voltage $\left(V_{\mathrm{DD}}\right)$ and the threshold voltage $\left(V_{\mathrm{T}}\right)$ is removed. Calibration can reduce uncertainty to \pm 0.015 FSR (Full Scale Range) in the range from $10 \mathrm{pF}$ to $100 \mathrm{pF}$ and to $\pm 0.008 \mathrm{FSR}$ in the range from $100 \mathrm{pF}$ to $1 \mathrm{nF}$ (Gaitán-Pitre et al., 2009).

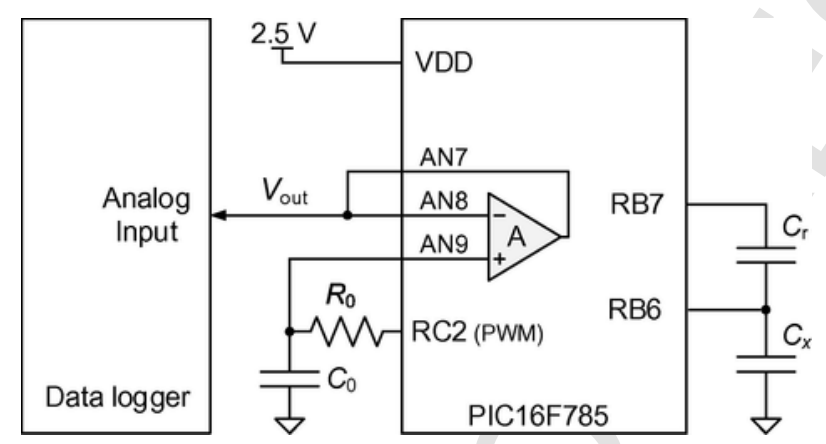

Fig. 4. Electronic interface circuit for the capacitive LWS $\left(C_{x}\right)$ and its connection to the analog input of a data logger. The function of the pins PX.0 and PX.1 in Fig. 3 were, respectively, implemented by pins RB6 and RB7 of the microcontroller.
The interface circuit designed can accept more capacitive sensors by using additional MCU I/O pins. If sensor capacitances are similar, they can share a single reference capacitor. Otherwise, the desired resolution can be achieved by using separate reference capacitors for different sensor ranges or for other capacitive sensors intended for additional parameters such as temperature and solar radiation.

The resulting digital value $N_{x}$ was used to modulate a PWM signal generated by the MCU. This signal was then low-pass filtered by an $R C$ circuit (Fig. 4) hence the resulting dc voltage, proportional to $C_{x}$ can be read externally, for example by a data logger. To avoid voltage loading effects, the low-pas filtered signal is buffered to yield $V_{\text {out }}$. This indirect digital-to-analog conversion allows a data logger to simultaneously measure the custom LWS and a commercial LWS with voltage output during our tests. Obviously, in normal use, the digital output of the MCU can be directly recorded or transmitted without this intermediate digital-to-analog conversion used for our experimental comparison with a commercial sensor that yields an analog output.

The power consumption of the measurement process depends on its duration hence on the resulting digital value $N_{x}$ and its maximum is $5 \mu \mathrm{W}$ for $18 \mathrm{~ms}$ when $N_{x}=255$.

\subsection{Experimental setup for performance assessment}

The performance of the two custom capacitive artificial leaves (RECT and OVAL) and the designed EIC have been compared against two commercial sensors, both in a controlled environment and in open-air conditions. The commercial sensors were: (i) the "rain sensor" (Telecontrolli Spa) (Fig. 5, left) built on a ceramic substrate (alumina) and which does not include any electronic interface, and (ii) the "LWS leaf wetness sensor" (Decagon Devices) (Fig. 5, right) built on a $0.65 \mathrm{~mm}$ thick fiberglass substrate and which includes its own electronic circuitry. Table 1 summarizes the shapes and dimensions of the two custom leaves and the two commercial LWSs, henceforth named Model A (rain sensor) and Model B (leaf wetness sensor).

\subsubsection{Indoors (lab) tests}

First, the capacitance of the three sensors with no electronics was measured with a 4294A impedance analyzer (Agilent Technologies) in dry and wet conditions. Sensor surfaces were homogeneously sprayed with water to obtain a $100 \%$ wet condition and later dried with a hair dryer.

Next, the transfer characteristic of the EIC shown in Fig. 4 with $R_{0}=100 \mathrm{k} \Omega$ and $C_{0}=10 \mu \mathrm{F}$ in the PWM demodulator was determined by measuring a set of known capacitors. $R_{0}$ and $C_{0}$ were chosen to obtain a PWM signal of $1 \mathrm{kHz}$. The test capacitors were selected to cover the sensor capacitance range observed in the first test, and $C_{\mathrm{r}}$ was accordingly selected.

Finally, the output voltages of the four sensors in dry and wet conditions were compared to each other.

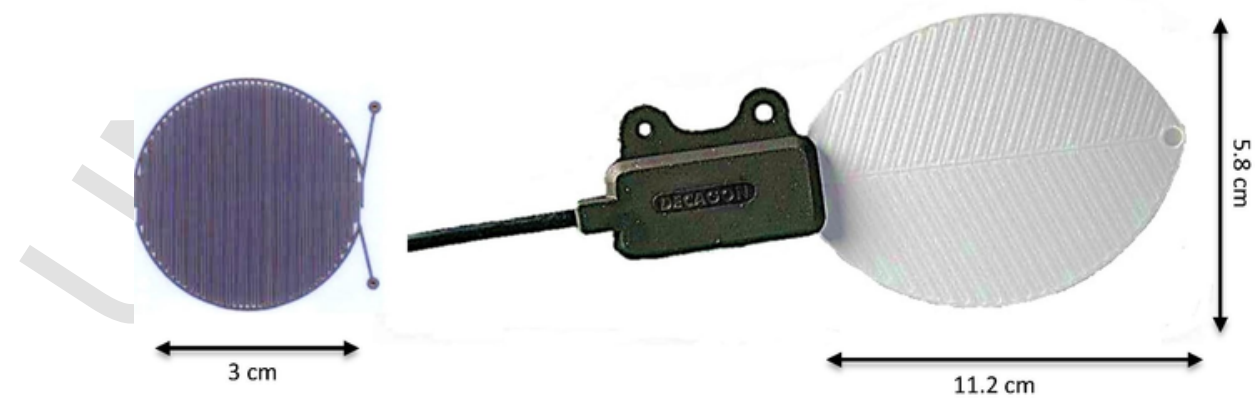

Fig. 5. Leaf wetness sensors: "Model A" from Telecontrolli (left) and "Model B" from Decagon Devices, with integrated electronics (right) (not to scale). 
Table 1

Dimensions (length $\times$ width $\times$ thickness) of the four leaf wetness sensors measured.

\begin{tabular}{lll}
\hline RECT & Rectangular & $4.5 \mathrm{~cm} \times 2.0 \mathrm{~cm} \times 1.6 \mathrm{~mm}$ \\
OVAL & Oval & $11.2 \mathrm{~cm} \times 5.8 \mathrm{~cm} \times 0.3 \mathrm{~cm}$ \\
Model A & Circular & $3.0 \mathrm{~cm} \times 3.0 \mathrm{~cm} \times 0.1 \mathrm{~cm}$ \\
Model B & Oval & $11.2 \mathrm{~cm} \times 5.8 \mathrm{~cm} \times 0.3 \mathrm{~cm}$ \\
\hline
\end{tabular}

\subsubsection{Outdoor validation tests}

The response of the OVAL, Model A and Model B sensors were compared through two outdoor tests: first, the two commercial sensors (Model A and Model B) and later the two oval-shaped sensors (OVAL and Model B). Both tests were performed in an outdoor terrace with the sensors spaced $20 \mathrm{~cm}$ and the same spatial orientation (Fig. 6) during two or more days. The output analog signals from the novel EIC and Model B's interface were simultaneously recorded by a commercial data logger (HOBO U12-013, Onset).

\section{Experimental results and discussion}

\subsection{Capacitance of dry and wet artificial leaves}

Table 2 summarizes the capacitance values measured for sensors RECT, OVAL and Model A in fully wet and dry conditions with the Agilent 4294A impedance analyzer, using an $R-C$ series equivalent impedance model. The relative uncertainty of the impedance analyzer is below $\pm 1 \%$ for capacitances from $10 \mathrm{pF}$ to $1 \mathrm{nF}$ when measuring at $100 \mathrm{kHz}$ and $0.5 \mathrm{~V}$ in the oscillator output level. Model B capacitance could not be measured because it cannot be separated from its electronic circuitry.

Model A shows the capacitance variation specified by the manufacturer. Capacitance in dry condition increases with leaf surface area (OVAL compared to RECT) and with the number of interdigitated electrodes (Model A compared to RECT), and relative capacitance changes are larger for small-capacitance sensors, as expected.

\subsection{Electronic interface circuit transfer characteristic}

The circuit in Fig. 4 was designed with $C_{\mathrm{r}}=15 \mathrm{nF}$ for the RECT sensor and $C_{\mathrm{r}}=33 \mathrm{nF}$ for OVAL and Model A sensors. These values were selected to guarantee $C_{\mathrm{r}}>>C_{x}$ in the wet condition (largest $C_{x}$ ) but without overflowing the microcontroller's counter register.

Since the relation between $N_{\mathrm{x}}$ and $C_{x}$ in Eq. (1) is hyperbolic, Fig. 7 shows the relation between $1 / C_{x}$ and $V_{\text {out }}$. Fig. 7 (left) shows $V_{\text {out }}$ for $C_{x}$ from $10 \mathrm{pF}$ to $90 \mathrm{pF}$, the range for the RECT sensor and Fig. 7 (right) shows $V_{\text {out }}$ for Model A's $C_{x}$ range (from $70 \mathrm{pF}$ to $400 \mathrm{pF}$ ). Test capacitors were measured with a model 4080D LCR meter (Motech Industries) at $10 \mathrm{kHz}$ (switching frequency of the charge-transfer capacitive sensing method), whose uncertainty is $\pm(0.5 \%$ reading $+1 \mathrm{pF})$ from $15.91 \mathrm{pF}$ to $159.1 \mathrm{pF}$, and $\pm(0.2 \%$ reading $+1 \mathrm{pF})$ from $159.1 \mathrm{pF}$ to $1.591 \mu \mathrm{F}$.

The relationship between $1 / C_{x}$ and $V_{\text {out }}$ is roughly linear. Linear regression yields the following equations for $V_{\text {out1 }}$ (RECT capacitance range) and $V_{\text {out2 }}$ (Model A capacitance range),

$$
\begin{aligned}
& V_{\text {out } 1}(\mathrm{~V})=0.27+33.62 \times 1 / C_{x} \quad(\mathrm{R}=0.993) \\
& V_{\text {out } 2}(\mathrm{~V})=0.05+112.17 \times 1 / C_{x} \quad(\mathrm{R}=0.999)
\end{aligned}
$$

from where the sensitivity and offset can be directly obtained. Table 3 summarizes them together with an estimate of the full-scale range (FSR) error for both reference capacitors $\left(C_{\mathrm{r}}=15 \mathrm{nF}\right.$ and $\left.C_{\mathrm{r}}=33 \mathrm{nF}\right)$.

One issue with the charge transfer approach used here is that the measurement resolution of $C_{x}$ is not uniform within the whole measurement range and worsens for the higher $C_{x}$ values (Gaitán-Pitre et al., 2009). From Eq. (1),

$$
\Delta C_{x}=\frac{C_{x}}{N_{x}-1}=\frac{C_{x}^{2}}{C_{r} \ln \left[\frac{V_{\mathrm{OH}}}{V_{\mathrm{OH}}-V_{T}}\right]-C_{x}}
$$

Therefore, the resolution will be better in dry conditions because $C_{\mathrm{x}}$ is smaller (larger $N_{x}$ ). Since the capacitance of the dry sensor is about 5 times smaller than in wet conditions (Table 2), the resolution will be about 25 times better which favor the detection of dew onset and dew dry off.

\subsection{Wet-dry tests of the EIC connected to different artificial leaves}

The three first rows of Table 4 show $V_{\text {out }}$ for the three different sensors connected to the EIC as a function of sensor wetness and the last row shows the voltage output of Model B. The RECT and OVAL sensors yield the highest output voltage hence provide a quite comfortable threshold to clearly differentiate between wet and dry conditions. Model A connected to the EIC also provides an output voltage higher than that of Model B.

The time evolution of $V_{\text {out }}$ for the wetting/drying test was obtained by simultaneously recording with the data logger the voltage response of Model A connected to the new EIC and that of Model B. Both sensors were placed in the same position and their surface wetness was increased with a sprayer and then dried with a hair dryer. Fig. 8 shows that both sensors roughly display the same evolution hence demonstrating that the proposed EIC allows Model A to yield the same qualitative information as Model B but with better sensitivity. (In Fig. 8 and the following figures, the sign of $V_{\text {out }}$ has been inverted and the graph has been vertically shifted to match $0 \mathrm{~V}$ in dry condition.) Nevertheless, the difference in size and materials makes Model B to dry quicker than Model A hence affecting the correlation coefficient $(R=0.89)$ between the two responses.

OVAL and Model B, which have similar shape and dimensions (Fig. 9) but are built from different materials, were also compared in the laboratory. The results from the same wetting-drying experiment are shown in Fig. 10. The response of OVAL closely resembles that of Model B and the correlation coefficient is now 0.98. However, OVAL is more sensitive because it yields a $1.3 \mathrm{~V}$ voltage change compared to $0.45 \mathrm{~V}$ change for Model B. There is a small time delay $(\Delta t<10 \mathrm{~s})$ be-
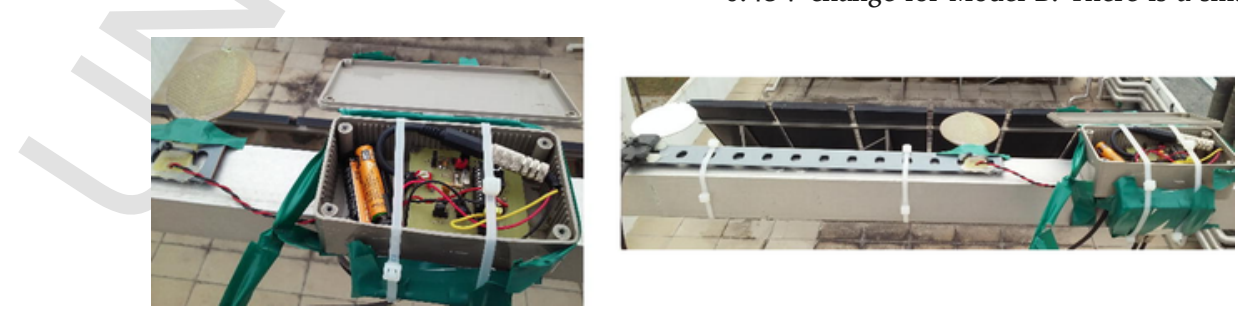

Fig. 6. Measurement setup for open-air tests. 
Table 2

Capacitance changes of RECT, OVAL and Model A artificial leaves.

\begin{tabular}{llll}
\hline Sensor & Dry $(\mathrm{pF})$ & $100 \%$ wet $(\mathrm{pF})$ & $\Delta C(\mathrm{pF})$ \\
\hline RECT & 15.2 & 90.0 & $\approx 75$ \\
OVAL & 70.2 & 381.3 & $\approx 310$ \\
Model A & 106.7 & 402.1 & $\approx 305$ \\
\hline
\end{tabular}

tween the two responses attributable to the latency of the communication between the data logger and the computer.

\subsection{Outdoors validation}

\subsubsection{Test 1: Model A sensor vs. Model B sensor}

We characterized the response of the EIC connected to the commercial circular sensor (Model A) and compared it to that of the commercial system (Model B) during two consecutive days.

Fig. 11 shows an extract of the results obtained (500 min of over 2000 min studied). The correlation coefficient of both responses is 0.85 meaning that both systems roughly yield the same qualitative information about leaf wetness in open-air conditions. Differences in shape, size and coating (Model A, circular, $3 \mathrm{~cm}$ in diameter, and Model B, OVAL, $11.2 \mathrm{~cm} \times 8 \mathrm{~cm}$ ) can explain the reduced correlation already seen in the lab tests.

\subsubsection{Test 2: OVAL sensor vs. Model B sensor}

Fig. 12 shows an excerpt of leaf wetness measurements performed with the OVAL (Fig. 12a) and Model B sensors (Fig. 12b) for three consecutive days. These results have been compared with rain recording (Fig. 12c) and the measured air temperature and (calculated) dew point temperature (Fig. 12d) from a nearby public weather station (Viladecans weather station). Dew appears when the condensation temperature equals the ambient temperature (Lawrence, 2005) and lasts for some time after ambient temperature is higher than condensation. The time delay between some events in the Viladecans weather station and the measurements on our sensors can be attributed to the $5 \mathrm{~km}$ that separate the two locations.

Measurements show that both sensors detect rain (event I and event II). Rain in event I $(0.1 \mathrm{~mm})$ is simultaneously detected by both sensors but the OVAL sensor is more sensitive $(\Delta V=0.8 \mathrm{~V})$ than Model B sensor $(\Delta V=0.1 \mathrm{~V})$, as already observed in the laboratory tests (Table $4)$. The correlation coefficient between the two responses is 0.92 . Rainfall at event II $(2 \mathrm{~mm})$, which occurs in a period of time when there is also condensation, is also simultaneously detected by both OVAL $(\Delta V=1.4 \mathrm{~V})$ and Model B $(\Delta V=0.5 \mathrm{~V})$ sensor. Since the rain was more intense than event I, the voltage values measured are the saturation values of both sensors (Table 4).

The OVAL sensor dries up much more slowly than Model B. For this reason the Model B sensor detects two dew points after rainfall (events III and IV), while OVAL sensor remains wet until the end of three events. For these reason, the OVAL sensor shows a larger wetness duration $(\Delta t=29 \mathrm{~h})$ than Model B $(\Delta t=12 \mathrm{~h})$. These results are summarized in Table 5 . These differences in the drying process could presumably be due to the different leaf coating used, which makes the correlation coefficient to fall down to 0.23 .

Therefore, the differences in shape, size, materials and surface treatments in open-air conditions become very important, particularly during the drying process. Environmental parameters such as wind direction and intensity and solar radiation are also relevant. The onset of rain can be correctly detected but the duration of rain episodes or leaf wetness resulting from them cannot be directly obtained from the raw signal, which is not a surprise as neither leaves from different plants nor leaves from the same plant dry off at the same time.

\section{Conclusions}

Plant disease cycles and epidemic development depend on foliar wetness but no proved method is available for in-field leaf wetness duration (LWD) measurement. This can be attributed to both the lack of an agreed definition for leaf wetness and to the different response of commercial leaf wetness sensors (LWSs). These sensors comprise an emulated leaf and, in some cases, an electronic interface that yields a voltage indicative of the amount of free water on the artificial leaf surface. Electronic interfaces provide a repetitive response but albeit the capacitive sensors tested change their impedance at the same time when wetted, they dry off at very different rates. This diverse response arises from their different shapes, sizes, materials and surface properties, in addition to inclination and orientation, and makes it very difficult to establish fixed thresholds for the wet-to-dry transition.

We have developed a novel electronic interface circuit (EIC) for artificial leaves based on capacitance changes that is more sensitive to leaf wetness than a commercial sensor with integrated electronics. Furthermore, capacitance is measured by using the charge-transfer method implemented by a microcontroller (MCU) without any additional active analog component. This results in a simple, compact solution whose components cost less than 5 USD yet this EIC still allows to determine duration intervals according to programmable thresholds and to communicate the results.

This EIC has been successfully applied to custom and commercial emulated leaves and can be adapted to any other capacitive sensor by changing the value of a single component hence it can be helpful in testing different leaf shapes and sizes, electrode configurations and sur-
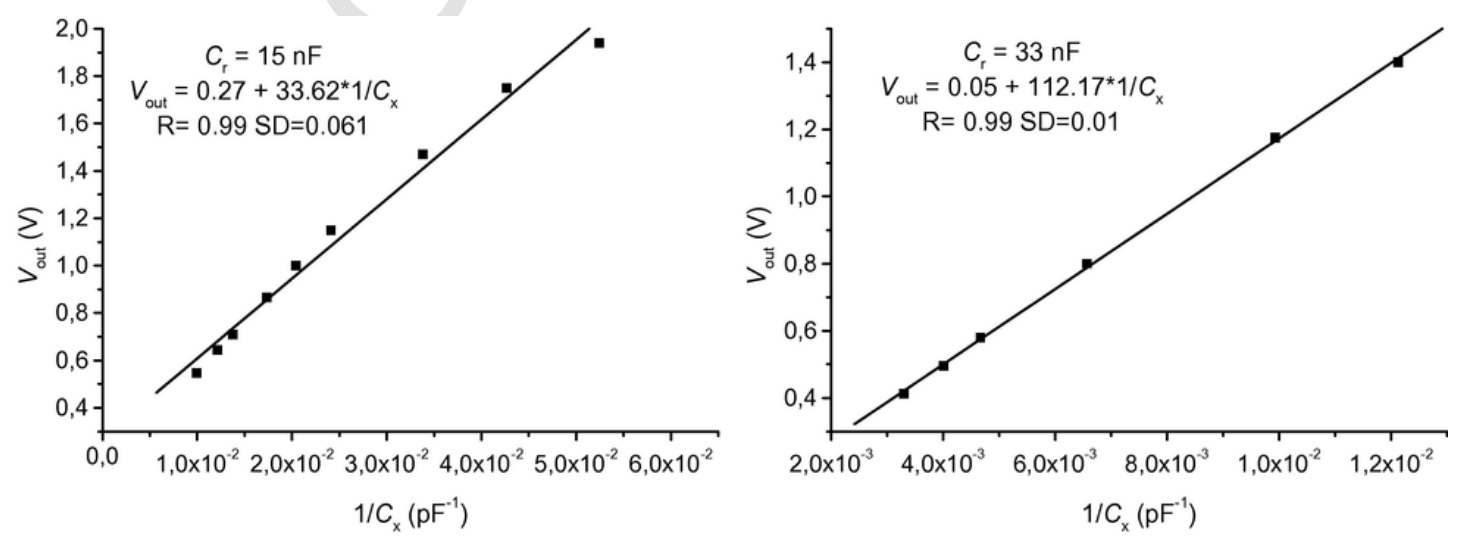

Fig. 7. Output voltage of the proposed EIC as a function of $1 / C_{x}$. Left: $C_{x}$ from $15 \mathrm{pF}$ to $80 \mathrm{pF}$ with $C_{\mathrm{r}}=15 \mathrm{nF}$. Right: $C_{x}$ from $70 \mathrm{pF}$ to $400 \mathrm{pF}$ with $C_{\mathrm{r}}=33 \mathrm{nF}$. 
Table 3

Characteristics of the EIC.

\begin{tabular}{|c|c|c|c|c|}
\hline & $\begin{array}{l}\text { Offset } \\
\text { (V) }\end{array}$ & $\begin{array}{l}\text { Sensitivity } \\
(\mathrm{V} / \mathrm{pF})\end{array}$ & $\begin{array}{l}\text { Residuals std. } \\
\text { dev. } \Delta V_{\text {out }} \\
(\mathrm{mV})\end{array}$ & $\begin{array}{l}\text { FSR error } \\
\Delta V_{\text {out }} / V_{\text {FSR }} \text {, } \\
(\%)\end{array}$ \\
\hline $\begin{array}{l}C_{\mathrm{r}}=15 \\
\mathrm{nF}\end{array}$ & 0.27 & 33.6 & \pm 96 & 5.4 \\
\hline $\begin{array}{l}C_{\mathrm{r}}=33 \\
\mathrm{nF}\end{array}$ & 0.05 & 112.2 & \pm 45 & 3.2 \\
\hline
\end{tabular}

Table 4

Response of the three artificial leaves connected to the electronic interface compared to model B LWS.

\begin{tabular}{lllll}
\hline Sensor & Dry $(\mathrm{V})$ & $100 \%$ wet $^{\mathrm{a}}(\mathrm{V})$ & $\Delta V(\mathrm{~V})$ & $S(\mathrm{~V} / \mathrm{mL})$ \\
\hline RECT & 0.0 & 1.5 & 1.5 & 1.5 \\
OVAL & 0.0 & 1.4 & 1.4 & 0.7 \\
Model A & 0.1 & 0.9 & 0.8 & 0.6 \\
Model B & 0.0 & 0.5 & 0.5 & 0.2
\end{tabular}

a $1 \mathrm{~mL}$ water for RECT and Model A sensors, and $2 \mathrm{~mL}$ water for OVAL and Model B sensors.

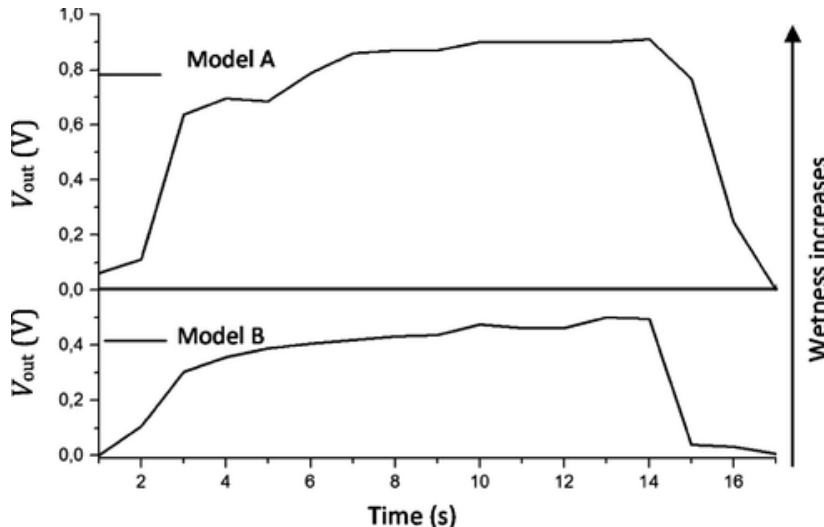

Fig. 8. Response of Model A and Model B sensors to a wetting-drying test in the laboratory.

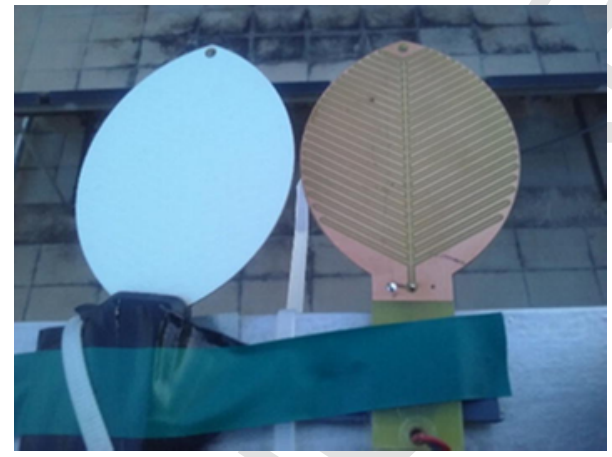

Fig. 9. Model B (left) and OVAL (right) sensors ready for the wetting-drying experiment

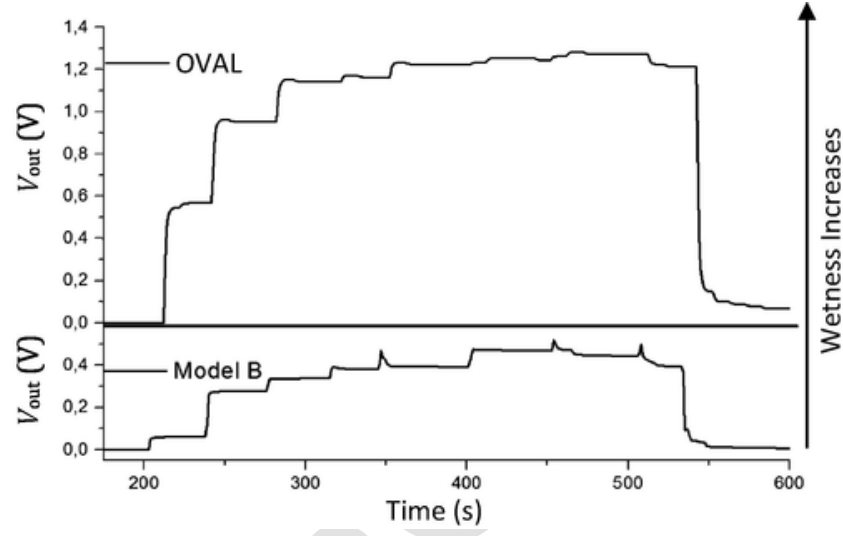

Fig. 10. Response of OVAL and Model B sensors to a wetting-drying test in the laboratory. The delay observed in the OVAL response is due to the latency of the communication with the computer.

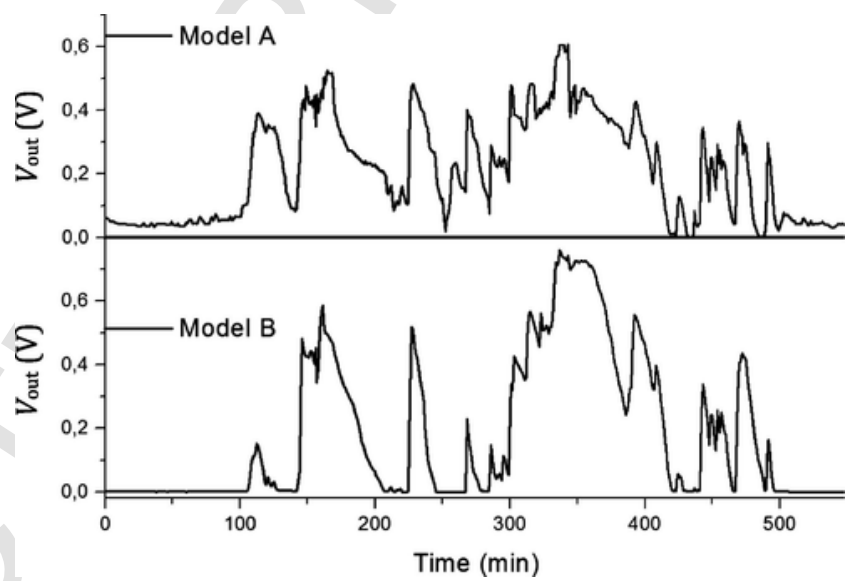

Fig. 11. Response of Model A and Model B LWSs during a $500 \mathrm{~min}$ ( $8 \mathrm{~h} 20 \mathrm{~min}$ ) extract of the $2000 \mathrm{~min}$ (33h $20 \mathrm{~min})$ test.

face layers that better emulate specific plant leaves. Its low cost opens up the possibility of simultaneously measuring several artificial leaves with different inclinations and orientations, and also measure other sensors, for example for temperature, wind or solar radiation.

\section{Uncited references}

\section{Acknowledgements}

The authors gratefully acknowledge the insightful comments of the reviewers. Also, the authors thank the Castelldefels School of Telecommunications and Aerospace Engineering (EETAC-UPC) for its research facilities and Mr. F. López for his technical support. 

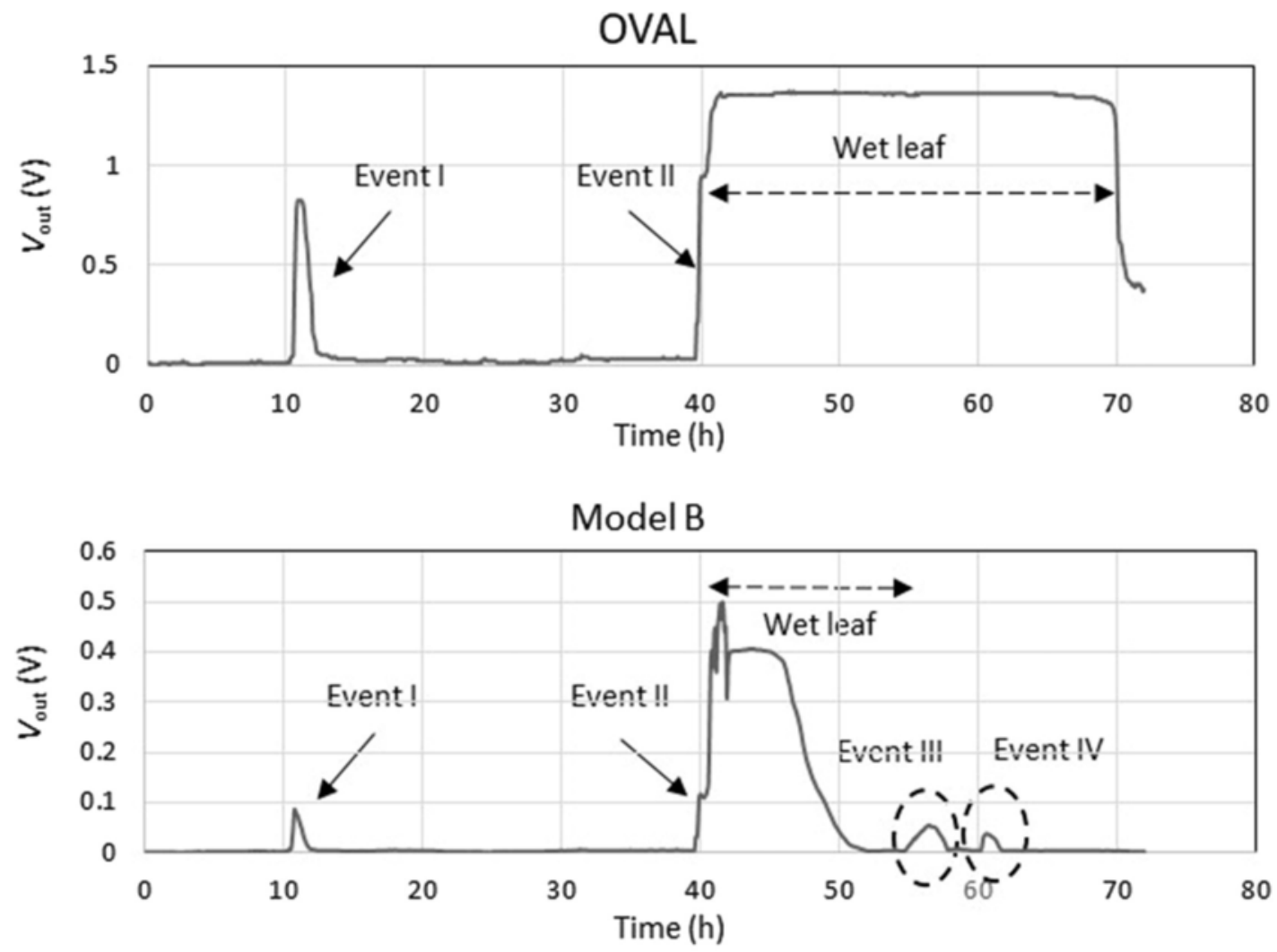

Rainfall

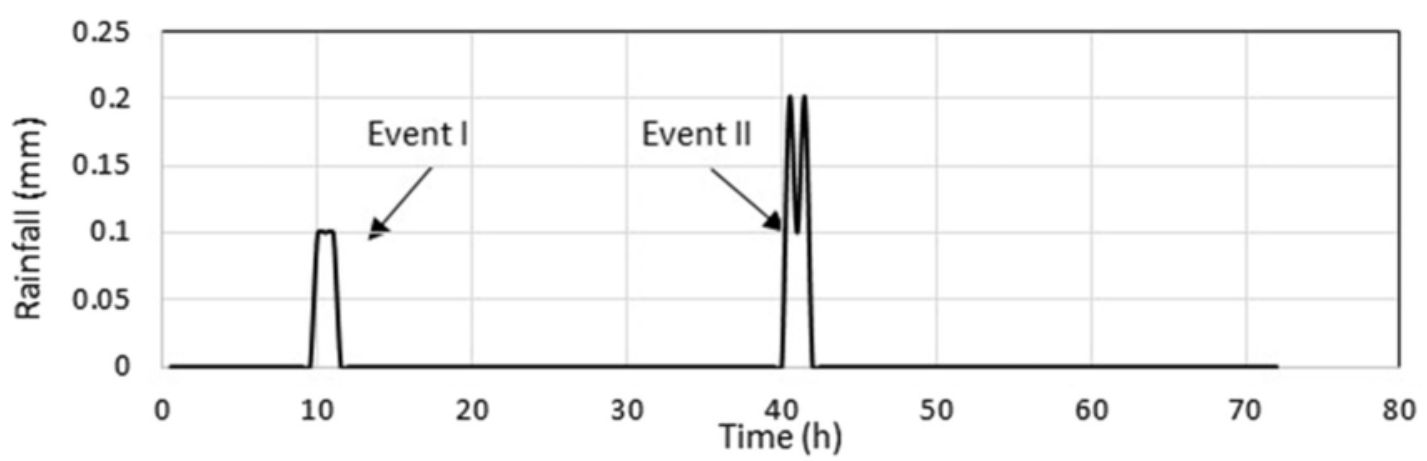

Temperature and Dew Point Temperature

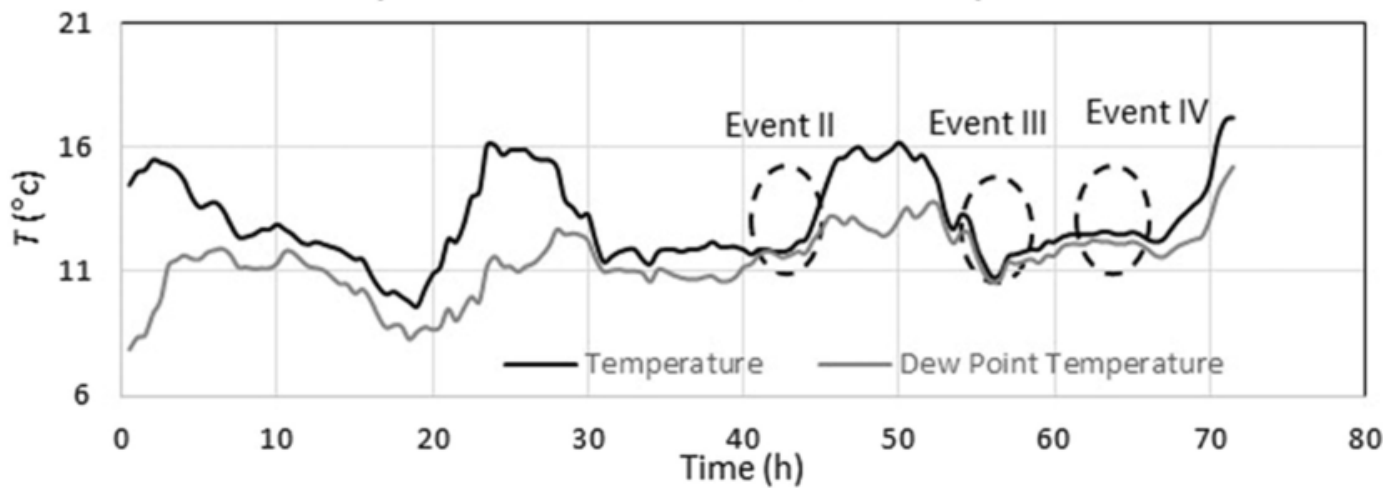

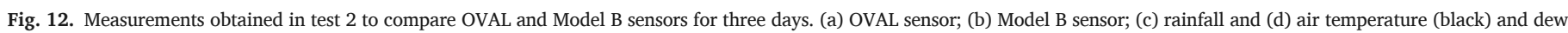
point temperature (gray) from a nearby public weather station. 
Table 5

Response of the OVAL and Model B LWSs to dew and rain presence and duration.

\begin{tabular}{lllll}
\hline Sensor & $\begin{array}{l}\text { Rain event I } \\
(0.1 \mathrm{~mm})\end{array}$ & \multicolumn{2}{l}{$\begin{array}{l}\text { Rain event II }(0.2 \mathrm{~mm}) \text { and Dew point } \\
\text { detection }\end{array}$} \\
\hline & $\begin{array}{l}\Delta V \\
(\mathrm{~V})\end{array}$ & $\begin{array}{l}\Delta t \\
(\mathrm{~h})\end{array}$ & $\Delta V(\mathrm{~V})$ & $\Delta t(\mathrm{~h})$ \\
\hline $\begin{array}{l}\text { OVAL } \\
\text { Model }\end{array}$ & 0.8 & 1 & 1.4 & 29 \\
B & 0.1 & 1 & 0.5 & 12 \\
\hline
\end{tabular}

\section{References}

Campbell Scientific. < https://s.campbellsci.com/documents/us/manuals/lws.pdf > (accessed April 19, 2017).

Davis, D.R., Hughes, J.E., 1970. A new approach to recording the wetting parameter by the use of electrical resistance sensors. Plant Dis. Report. 54, 474-479.

Davis Instruments. < http://www.davisnet.com/ > (accessed April 19, 2017).

Decagon Devices. <http://www.decagon.com/en/canopy/canopy-measurements/ lws-leaf-wetness-sensor/ > (accessed April 19, 2017).

Gaitán-Pitre, J., Gasulla, M., Pallàs-Areny, R., 2009. Analysis of a direct interface circuit for capacitive sensors. IEEE Trans. Instrum. Meas. 58, 2931-2937.

Getz, R.R., 1992. Report on the measurement of leaf wetness. WMO (Commission for Instruments and Methods of Observation).

Gillespie, T.J., Kidd, G.E., 1978. Sensing duration of moisture retention using electrical impedance grids. Can. J. Plant Sci. 54, 179-187.

Lau, Y.F., Gleason, M.F., Zriba, N., Taylor, S.E., Hinz, P.N., 2000. Effects of coating, deployment angle, and compass orientation on performance of electronic wetness sensors during dew periods. Plant Dis. 84, 192-197.
Lawrence, M.G., 2005. The relationship between relative humidity and the dew point temperature in most air: a simple conversion and applications. Bull. Am. Meteorol. Soc. $86,225-233$

Lierop, P.V., Lindquist, E., Sathyapala, S., Franceschini, G., 2015. Global forest area disturbance from fire, insect pests, diseases and severe weather events. For. Ecol. Manage. 352, 78-88.

Madeira, A.C., Kim, K.S., Taylor, S.E., Gleason, M.L., 2002. A simple cloud-based energy balance model to estimate dew. Agric. For. Meteorol. 111, 55-63.

Mamishev, A.V., Sundara-Rajan, K., Yang, F., Du, Y., Zahn, M., 2004. Interdigital sensors and transducers. Proc. IEEE 92, 808-845.

Mashonjowa, 2013. Estimation of leaf wetness duration for greenhouse roses using a dynamic greenhouse climate model in Zimbabwe. Comput. Electr. Agric. 95, 70-81.

Microchip Technology. < http://ww1.microchip.com/downloads/en/DeviceDoc/41249a. pdf $>$ (accessed April 19, 2017).

Motech Industries, Inc. < $<$ https://webbuilder.asiannet.com/ftp/810 MT\%204080\%20User\%20Manual.pdf > (accessed April 19, 2017).

Onset. <http://www.onsetcomp.com/products/data-loggers/u12-013> (accessed 19 April 2017).

Philipp, H., 1999. Charge transfer sensing. Sens. Rev. 19 (2), 96-105.

Rowlandson, T., Gleason, M., Sentelhas, P., Gillespie, T., Thomas, C., Hornbuckle, B. 2015. Reconsidering leaf wetness duration determination for plant disease management. Plant Dis. 99, 310-319.

Schmitz, H., Grant, R., 2009. Precipitation and dew in a soybean canopy: spatial variations in leaf wetness and implications for Phakopsora pachyrhizi infection. Agric. For. Meteorol. 149, 1621-1627.

Sentelhas, P.C., Gillespie, T.J., Gleasonc, M.L., Monteiro, J.E.B.A., Helland, S.T., 2004. Operational exposure of leaf wetness sensors. Agric. Forest Meteorol. 126, 59-72.

Telecontrolli. < www.telecontrolli.com > (accessed April 19, 2017)

Viladecans weather station. < http://www.meteo.cat/observacions/xema/ dades? codi $=$ UG $>$ (accessed September 8, 2017)

Wichink Kruit, R.J.W., van Paul, A.J., Jacobs, A.F.G., Heusinkveld, B.G., Comparison between four methods to estimate leaf wetness duration caused by dew on grassland. In: 26th Conference on Agricultural and Forest Meteorology, 23-27 August 2004. American Meteorological Society, Vancouver, BC, Canada. 